\title{
UMBRALES
}

\section{Gamificación para la Educación Intercultural}

\author{
Umbrales: Gamification for Intercultural Education
}

\author{
VANESSA FONSECA
}

Universidad de Costa Rica, Costa Rica

\begin{tabular}{l} 
KEY WORDS \\
\hline Gamification \\
Intercultural education \\
Transmedia \\
Boruca culture \\
Decolonial pedagogy
\end{tabular}

PALABRAS CLAVE

Gamificación

Educación intercultural

Transmedia

Cultura boruca

Pedagogía decolonial

\begin{abstract}
Umbrales is a transmedia proposal that seeks to invite primary school audiences or museum audiences to learn about different aspects (lexicons, material culture, oral culture, ethnobotany) of the Boruca culture, an indigenous community located in the South Pacific of Costa Rica. The project " Umbrales: A transmedia strategy for intercultural education" consists of different media platforms such as a virtual world in Unity $3 D, a$ brochure entitled "The Treasures of Caño Island", which shows elements of paper engineering and augmented reality, an interactive house in Second Life and a parallax website where the narratives of the other mentioned instances are complemented with pedagogical guides and creativenarrative-performative workshop-type activities.
\end{abstract}

\section{RESUMEN}

Umbrales, es una propuesta transmedia que buscar invitar a audiencias de educación primaria o públicos de museos a conocer diferentes aspectos (léxicos, cultura material, cultura oral, etnobotánica) de la cultura boruca, comunidad indígena localizada en el Pacífico Sur de Costa Rica. El proyecto "Umbrales: Una estrategia transmedia para la educación intercultural" consta de diferentes plataformas mediáticas tales como un mundo virtual en Unity 3D, un folleto titulado "Los tesoros de la Isla del Caño", que muestra elementos de ingeniería de papel y realidad aumentada, una casa interactiva en Second Life y un sitio web parallax donde se complementan las narrativas de las otras instancias mencionadas con guías pedagógicas y actividades tipo taller creativonarrativo-performativo.

Recibido: 20/01/2020

Aceptado: 10/05/2020

\section{GLOBAL牦) KNOWLEDGE




\section{Introducción}

$U$ mbrales, es una propuesta transmedia que buscar invitar a audiencias de educación primaria o públicos de museos a conocer diferentes aspectos (léxicos, cultura material, cultura oral, etnobotánica) de la cultura boruca, comunidad indígena localizada en el Pacífico Sur de Costa Rica.

El proyecto "Umbrales: Una estrategia transmedia para la educación intercultural" consta de diferentes plataformas mediáticas tales como un mundo virtual en Unity 3D, un folleto titulado "Los tesoros de la Isla del Caño", que muestra elementos de ingeniería de papel y realidad aumentada, una casa interactiva en Second Life y un sitio web parallax donde se complementan las narrativas de las otras instancias mencionadas con guías pedagógicas y actividades tipo taller creativo-narrativoperformativo.

Los usuarios transitan de una plataforma a la otra para aprender diferentes aspectos de la cultura boruca. El proyecto incluye una serie de misiones y retos que adentran al usuario en una experiencia interactiva que los hace deambular por diferentes pasajes o umbrales temporales y espaciales que se enriquecen con rompecabezas, animaciones o videos en realidad aumentada, mundos virtuales donde se ponen en práctica diversas técnicas que promueven un aprendizaje crítico basado en la resolución de problemas.

Umbrales ha sido producido por Vanessa Fonseca González y José Luis Arce Sanabria ambos profesores de la Escuela de Ciencias de la Comunicación Colectiva de la Universidad de Costa Rica en conjunto con un grupo transdisciplinario que incluye artistas de la comunidad boruca, antropólogos, comunicadores, lingüistas, diseñadores gráficos, entre otros.

\section{Perspectivas Teóricas}

Desde tiempos coloniales, el legado de los pueblos originarios de América ha sido sistemáticamente silenciado y desestimado por grupos hegemónicos a lo largo del continente. Esta desafortunada invisibilización no solo ha impactado negativamente la autoestima y la autoeficiencia de las comunidades originarias, sino que ha naturalizado una colonialidad del poder, una matriz dominante tripartita que también incluye la colonialidad del ser y del saber (Quijano, 1992; Mignolo, 2010; Walsh, 2010).

Gracias a esta matriz, el poder hegemónico colonial ejerció y legitimó el control y la dominación sobre los territorios conquistados sobre los que imponía una supuesta superioridad moral y conocimiento, en fin una misión "civilizatoria". Europa se convirtió en el modelo cultural, la única vía de llegar a la civilización. Una vez en el poder, el colonizador generó o produjo conocimiento sobre los pueblos colonizados, usualmente invisibilizando sus logros, su legado, demonizando sus prácticas culturales y sobre todo, prohibiendo la reproducción de su patrimonio cultural material e intangible: sus lenguas, sus dioses, sus historias, sus objetos, sus alimentos. El objetivo claramente era desarticular espacios y prácticas culturales necesarias para la reprodución y sobrevivencia de sus herencias culturales ancestrales, para así dejar libre el espacio para que la cultura del conquistador las suplantara.

De ahí que la colonialidad del poder no solo supone la conquista de territorios y recursos, sino que también implica la puesta en marcha de procesos de sujeción y subalternización orientados a producir subjetividades sumisas que no le ofrezcan oposición ni cuestionamientos (la colonialidad del ser). Esta lógica generó largos y dolorosos procesos en los que el sujeto colonial se vio tensado entre dos opuestos, la cultura originaria dominada y conquistada y el nuevo orden colonizador y "civilizador". Esta tensión lo obligaba a olvidar y silenciar sus tradiciones para acomodarse al nuevo orden y asimilarse a sus principios e ideología, el modo de ser del conquistador. En otras palabras, como miembros que sufrieron violentos procesos de colonización, ambos, tanto los que descienden de las comunidades originarias de América, así como los que descienden de los conquistadores, se encuentran en el dilema del sujeto colonial, como sujeto al borde, en crisis frente a dos identidades que ya lo niegan o lo rechazan.

Umbrales se inspira en los lineamientos propuestos por Catherine Walsh y Walter Mignolo (2019) o Tubino (2005) orientados a la implementación de una educación intercultural crítica. Sin embargo, reúne una variada gama de 
perspectivas teóricas y metodológicas. Se alínea con las preocupaciones externadas las perspectivas de pedagogías decoloniales y las denominadas epistemologías del Sur (De Sousa Santos y Meneses, 2014; De Sousa Santos, 2009) y la urgencia de propuestas que consideren los actuales retos que enfrentan las sociedades latinoamericanas en las culturas de convergencia y en la nueva ecología de los medios y las narrativas transmedia (Scolari, 2013, 2015, 2018). Apuesta al alfabetismo transmedial liderado por organizaciones como el Immersive Education Initiative y el Transmedia Literacy Project, como una opción hacia la inclusión digital de fundamental importancia en la activación de la agencia cultural de los pueblos originarios de América y la gestión de sus recursos materiales y patrimoniales tanto tangibles como intangibles.

A pesar de 500 años de colonización, la memoria y el patrimonio cultural siguen siendo la base de los grupos originarios latinoamericanos, quienes aún luchan entre la tradición y la modernización. Las comunidades boruca localizadas principalmente en dos ubicaciones del Pacífico Sur costarricense, Boruca y Curré, son espacios donde se manifiestan estas tensiones: cómo preservar su patrimonio, tradiciones orales, valores $\mathrm{y}$ prácticas culturales al mismo tiempo que se insertan en las demandas de un capitalismo y occidentalismo que los ha excluido sistemáticamente. Nuevas generaciones de borucas miran más allá de sus localidades para lograr educarse y trabajar, las formas tradicionales de vida no necesariamente constituyen hoy caminos viables de progreso y sobrevivencia. Considerando lo anterior, este proyecto intenta involucrar a generaciones jóvenes de borucas y a otros niños costarricenses o visitantes de museos en una experiencia transmedia en la que puedan aprender sobre el legado cultural y material de estas comunidades originarias y la relevancia de su aporte a una sociedad donde cada vez son más necesarios espacios y prácticas de educación crítica intercultural

Catherine Walsh $(2014,43)$ propone el pensamiento decolonial como una forma de desaprender: "To unlearn everything imposed by the colonization and dehumanization process in order to relearn how to become men and women." Parafraseando sus palabras, la decolonización surge cuando individuos o colectividades participan en una superación de la colonialidad del poder, cuando aquellos que han sido oprimidos por centurias se despiertan a una nueva consciencia. La escritora argumenta que es necesario distinguir interculturalidad funcional de interculturalidad crítica. La primera responde a las necesidades e intereses de instituciones sociales para el diálogo y el reconocimiento de la diferencia cultural, sin cuestionar las causas históricas y actuales de la opresión y la subalterización de los pueblos originarios. La segunda se produce desde y en el interior de comunidades subjugadas, quienes han sufrido opresión y subalterización y busca, más allá del diálogo y el reconocimiento de la diferencia cultural, las posibilidades de reinvención y reinserción ciudadana que la agencia cultural les ofrece (Walsh, 2009). Una forma de alcanzar lo propuesto por Walsh es promover prácticas en las que la agencia cultural permita a los individuos cuestionar el estado actual de sus condiciones materiales y revisitar eventos presentes o pasados desde una perspectiva crítica. Baker define el concepto de agencia de la siguiente manera:

The concept of agency can be understood to mark the socially determined capability to act and to make a difference. Agency has commonly been associated with notions of freedom, free will, acting, creativity, originality and the possibility of change brought about through the actions of sovereign individuals (...). Of course, precisely because socially constructed agency involves differentially distributed social resources that give rise to various degrees of the ability to act in specific spaces, so some actors have more scope for action than do others. (Baker, 2004, 4)

Al involucrar a comunidades boruca y no boruca en una experencia transmedia, este proyecto espera ofrecerles vías para re-leer sus experiencias culturales identitarias y crear sus propios contenidos-narraciones o producir espacios alternativos de agencia cultural mediante la transferencia tecnológica que 
proyecte sus propias habilidades y posibilidades creadoras. Las tecnologías inmersivas son herramientas potenciales para motivar a las audiencias jóvenes a generar productos culturales que vayan más allá de un taller, una clase formal tradicional o la visita a un museo. Pueden producir artefactos culturales que no solo promuevan la agencia cultural al interior de las comunidades étnicas originarias de América, sino ser espacios donde se generen prácticas de interculturalidad crítica para nuevas generaciones de costarricenses $u$ otros grupos interesados en el legado de los pueblos originarios de América Central.

La utilización de tecnologías educativas innovadoras para el desarrollo de proyectos de educación intercultural es una apuesta a formatos novedosos que generan más interacción con las audiencias jóvenes (Lanham, 2017). Ofrecen, además de una experiencia estética, lúdica y educativa, la posibilidad de que los usuarios se conviertan en colaboradores o coproductores, es decir, que la misma narrativa transmedia los invita a crear sus propias historias para que enriquezcan las propuestas iniciales (videojuego, folleto, casa interactiva, galería en sitio web parallax) y abren la posibilidad de que el mundo narrativo inicial se expanda exponencialmente.

En Latinoamérica sufrimos las paradojas de procesos de modernización donde ha estado ausente la modernidad como paradigma económico o cultural. Por muchas décadas los privilegios de los servicios eléctricos o de telefonía o acceso a internet no fueron del alcance de todos. Sin embargo, paradójicamente, los procesos de globalización de los últimos años le abrieron paso a procesos de modernización que se saltaron la modernidad. Hoy hay quienes disfrutan de internet $4 \mathrm{G}$ y nunca tuvieron acceso a telefonía fija. Los hay que pueden descargar contenidos de Netflix, pero antes nunca tuvieron televisión digital por cable. Según el GSMA (Global System for Mobile Communications) en Costa Rica hay 178 líneas activas por cada 100 habitantes (Montero, 2019). Costa Rica (178\%), el Salvador (159\%) y Uruguay (155\%) lideran la lista de países con mayor penetración de tarjetas SIMS en América Latina y superan a países como España (130\%) uno de los países con más penetración de tarjetas SIMS en Europa. La siguiente sección discute las razones por las que el proyecto Umbrales optó por visibilizar el legado de las comunidades boruca en esta propuesta transmedia.

\section{¿Por qué los Boruca?}

Históricamente lo que se conoce como Costa Rica ha sido un corredor o puente biológico y cultural habitado por diferentes grupos humanos pero, principalmente, caracterizado desde dos grandes regiones culturales: La región Chibcha al sur del país que la conecta con el norte del continente suramericano y la Gran Nicoya al norte que puede considerarse la frontera cultural sur de la región Mesoamericana. A lo largo del territorio costarricense se pueden encontrar descendientes de los ocho grupos originarios: cabécares, bri-bris, boruca o bruncas, chorotegas, huetares, maleku, térraba o teribe y ngöbes-bugle. Los boruca están localizados en la región de Pacífico Sur, especialmente en dos comunidades: Boruca y Curré. Junto a otros grupos indígenas originarios, se cree que los boruca migraron desde el norte de Suramérica y Panamá para habitar finalmente en el Valle del Diquis (su etimología en lengua boruca es (Dí $>$ > río y Crí $=$ grande), región de una inmensa riqueza en fauna, flora, sitios arqueológicos y un imponente paisaje dominado por varios ríos de abundante caudal, entre ellos, el majestuoso Río Grande de Térraba, que nace en las montañas tierra adentro y desemboca en el Oceáno Pacífico Sur.

En su recorrido crea deltas, irriga densos bosques tropicales que son el hábitat de muchas especies animales desde jaguares a guacamayas escarlata, desde monos hasta cocodrilos. Los antropólogos y arqueólogos consideran a los boruca como un "pueblo del agua", dada la importancia que el en Gran Rí tiene en su vida cotidiana, en sus rutas comerciales $y$ principalmente en sus historias orales y su cultura material. Más aún, el Parque de las Esferas ubicado en el Valle del Diquis, regado por el Río Grande de Térraba fue reconocido por Unesco como Patrimonio de la Humanidad en el 2014 y existen en la zona petrogliflos asociados a cursos, nacimientos y depósitos de agua. Este conjunto arqueológico que guarda gran cantidad de esferas de granito de diferentes dimensiones 
cuyo significado sigue siendo un misterio, hoy conforma un parque arqueológico protegido por su "Excepcional valor cultural, social, educativo y científico" y lo consituyen 4 sitios arqueológicos: Finca 6, Batambal, el Silencio y Grijalba 2 (https://www.gobiernolocalosa.go.cr/turismo/i nfo.php?item $=55$ ).

A pesar de 500 años de dominación cultural, los boruca han permanecido fieles a sus tradiciones y rituales. Desde tiempos coloniales eran temidos como aguerridos guerreros, que resistieron con valentía la conquista y la colonización. Hay registros etnológicos y de historias orales (Stone, 1949; Quesada, 1996; Morales, 1999; Fonseca, 2012; Constenla, 2011) que indican que los boruca navegaban desde tierra adentro hasta varias islas de los deltas que conforma el Grande de Térraba y hasta llegar a adentrarse en el Oceáno Pacífico Sur, específicamente a las islas del Caño y Violín (aproximadamente $19 \mathrm{~km}$ de navegación marítima). En estos viajes se proveían de diferentes recursos como alimentos, peces, mariscos, mieles silvestres y extraían en la costa sal de los troncos en la playa y un tinte natural sumamente valioso. La extracción del múrice, en boruca Surem o surem-is (Plicopurpura patula subsp. Pansa) y Plicopurpura columellaris (Quintanilla, 2012, 2014) la hacen hasta hoy de forma completamente sostenible y son uno de los pocos grupos indígenas que usa este molusco para teñir sus textiles, principalmente el algodón, sin matarlo en el proceso de extracción.

Para los Borucas una de las principales tradiciones es la Fiesta de Los Diablitos. Esta danza, representa simbólicamente la resistencia boruca frente a los conquistadores españoles. Los Diablitos representan a guerreros boruca que enmascarados enfrentan al Toro, que a su vez, representa al colonizador. Los Diablitos bailan junto al Toro hasta matarlo simbólicamente como muestra de su sobrevivencia y victoria. Esta celebración es ya conocida internacionalmente y cada día más turistas la visitan. En fin, los boruca ofrecen un patrimonio riquísimo que se manifiesta no solo en su tradiciones y legados culturales sino que pervive hasta nuestros días en recetarios (Morales, 1999), máscaras, tejidos, cestería y otras artesanías de gran trascendencia cultural.

\section{Metodología}

Este proyecto supuso ante todo una metodología cualitativa de consulta y trabajo con expertos en diferentes saberes. Se procuró un desenvolvimiento horizontal que integrara diferentes tipos de saber, actores, habilidades y competencias. De esta forma, se conformaron duplas creativas y de expertos que combinaron especialistas en antropología, lingüística, comunicación, diseño, programación e ingeniería de papel, entre otras.

En un primer paso se identificaron aspectos de cultura material, patrimonio, historia oral, educación ambiental. Con estas variables se definió que entre las tradiciones del pueblo boruca había elementos que calificaban y a partir de los cuales se podían definir un guión académico que permitiera el desarrollo de un proyecto creativo cuya meta era la elaboración diferentes materiales educativos que incluyeran animación 3D, espacios inmersivos, un folleto con una narrativa ilustrada tradicional, pero enriquecida con elementos digitales tales como videos sobre vida submarina, realidad aumentada y elementos en ingeniería de papel.

Se elaboraron los guiones académicos con base en investigaciones desarrolladas desde diferentes disciplinas ya publicadas en revistas o como libros académicos. De ahí se seleccionaron los contenidos que permitieran elaborar una narrativa que diera cuenta de los aportes de la comunidad boruca, su patrimonio intagible, su cultura material, sus tradiciones orales y la relevancia de estos legados en un horizonte de alfabetización intercultural digital en una cultura cada vez más convergente donde es necesario utilizar estrategias de alfabetización transmedia desde una perspectiva decolonial, es decir crítica.

Una vez elaborados los guiones académicos se validaron con expertos al menos en tres niveles diferentes de saber: Primero con miembros de la comunidad boruca, artistas y gestores culturales, segundo con expertos desde las diferentes disciplinas involucradas (tecnologías de la educación, antropología, comunicación y diseño, lingüística, entre otras). Finalmente, se consultó con usuarios potenciales de estos materiales educativos que, además de poder ser utilizados como tecnologías educativas novedosas, también 
pueden ser productos de promoción cultural y gestión turística rural.

Se desarrolló una maqueta o dummie que permitió hacer las validaciones mencionadas y la cual se está en proceso de presentación a autoridades del Sistema de Museos del Banco Central de Costa Rica y a personas clave en la gestión cultural del pueblo boruca para que constituyan un modelo o piloto de gestión de transferencia tecnológica, co-producción de contenidos con los usuarios y, en el mejor de los escenarios, de futura colaboración para la elaboración de otros materiales asociados a los presentes: el videojuego Umbrales, el folleto "Los tesoros de la isla del Caño", así como las actividades o guías didácticas propuestas ofrecen acercarse a este proyecto desde diferentes actividades creativas en las que los usuarios parten de la experiencia del videojuego, la lectura del folleto y la experimentación con sus diferentes recursos que se detallarán más adelante.

Este proyecto se basa en una perspectiva constructivista de los procesos de enseñanzaaprendizaje. En este sentido está abocado a diseñar experiencias educativas que sirvan para que el discente sea capaz de descubrir caminos no isomórficos para generar su propio aprendizaje. Es decir, se ha pensado en diseñar diferentes estímulos que permitan a los participantes descubrir desde sus propias experiencias, memorias y recursos, nuevos conocimientos o asociar lo anteriormente aprendido con contenido novedoso o desconocido.

Cuatro ejes fundamentan esta perspectiva constructivista que a la vez se entremezcla directamente con la estrategia metodológica: 1 . Generar materiales educativos novedosos, estimulantes y variados que permitan un intercambio rico entre los participantes y el facilitador. 2. La perspectiva de que el proceso de aprendizaje depende en gran medida de la participación, activa y comprometida del discente. 3. Favorecer intercambios productivos y de comunicación efectiva entre el facilitadordocente y los participantes. 4. Establecer dinámicas de colaboración que faciliten el proceso de enseñanza -aprendizaje entre los participantes.
De ahí que sea de particular importancia articular la perspectiva constructivista con el enfoque neurodidáctico, en particular la propuesta metodológica de Roberto Rosler y los siete pasos que propone para consolidar el aprendizaje.

a. Abrir las memorias sensoriales: En la activación de varios sentidos está la clave de la mayor recordación de los contenidos. Umbrales está diseñado pensando en provocar la curiosidad, busca la atención desde lo visual, auditivo, lo sorprendente. Las memorias sensoriales relevantes de los estudiantes intervienen como refuerzos a las conexiones neuronales que queremos forjar. Así, por ejemplo, utilizar elementos como la Fiesta de Los Diablitos, máscaras, lengua boruca con niños boruca es relevante como estrategia de aprendizaje pues relaciona conocimientos previos con lo experimentado a través del estímulo sensorial.

b. Generar reflexión. La historia de Umbrales está diseñada para ser pensada, para reflexionar sobre un estímulo parcial que se le ofrece al participante. Mediante una mayéutica socrática se trabaja para hacer un aprendizaje significativo. Entre una actividad $y$ otra se pueden introducir espacios de reflexión para que se establezcan las conexiones entre lo novedoso y lo conocido.

c. Hacerlo Propio: Entre el juego 3D Umbrales y la lectura de la historia "Los Tesoros de la Isla del Caño" pueden pasar varios días. Hay materiales educativos alternativos como juguetes de papel que les sirven a los estudiantes para crear sus propios contenidos con lo ya aprendido. De igual manera se puede usar el role playing ya que las historias se puede jugar varias veces dando respuesta o ensayando diferentes finales en cada ocasión para reforzar lo aprendido.

d. Retroalimentación: Se pueden establecer dinámicas de tríos o dúos para verificar que el conocimiento se está asentando en la mente de los participantes Trivias, Kahoots con un solo objetivo de aprendizaje permiten medir si ha habido aprendizaje o no. También se pueden establecer dinámicas de 
discusión como debates, paneles, etc. Es importante que el feedback o retroalimentación esté asociado con alguna gratificación (premios, concursos, estrategias gamificadas) para que se consolide el circuito neurológico de la motivación.

e. Practicar: Se ofrece al participante apropiarse de los conocimientos generando sus propias historias-narrativas con elementos ya conocidos. Se les brinda a los participantes diferentes plataformas de creación de juegos de rol ya sean digitales o tradicionales : teatros de sombra, títeres tradicionales, juguetes de papel, Minecraft, CoSpaces, creación de contenidos 360 en hyperlapse, mapas culturales con el fin de poner en acción lo aprendido

f. Repensar: Una vez formulados los contenidos y actividades anteriores se revisitan con los participantes buscando su mirada crítica ante el estímulo ofrecido para así poder pasar de la memoria de corto plazo a la memoria de largo plazo y utilizar la memoria de trabajo. ¿Cómo se podrían mejorar esas historias, aplicaciones, cuentos, role playing, minijuegos? Incentivar la creatividad más allá de lo imaginado hasta ahora.

g. Recuperar: En esta etapa final lo que interesa es resituar el conocimiento aprendido de forma que seamos capaces de recuperarlo mediante pistas, acertijos, de ser posible establecer un misterio por resolver en la clase tipo "escape room" donde los participantes activen lo aprendido y les sea de utilidad para resolver esa nueva situación que enfrentan con el conocimiento obtenido de todo el proceso anterior.

El presente proyecto viene a complementar los esfuerzos y recursos de un proyecto anterior denominado "Museo Inmersivo de Culturas Antiguas de Costa Rica" (No 724-B3-037). El objetivo principal fue la creación de una serie de espacios virtuales inmersivos que le permitieran a los usuarios aprender-jugando sobre la historia y el patrimonio cultural del pueblo boruca. Este proyecto recibió el Fondo de Estímulo a la Investigación en el $2014 \quad(\$ 10,000)$ de la Universidad de Costa Rica con el cual se ha podido desarrollar la plataforma inmersiva
Umbrales y las diferentes misiones que le facilitan al usuario(a) un escenario interactivo donde se relaciona con personajes, objetos y ambientes que le permiten aprender sobre la historia y el patrimonio cultural boruca.

Umbrales también se apropia del Aprendizaje Basado en Problemas (ABP). Esta metodología se centra en el estudiante quien adquiere conocimientos, habilidades y actitudes al enfrentar y resolver problemas o retos que enfrenta en su vida cotidiana, se trate de situaciones de enseñanza-aprendizaje formal (aula) o no formal (museos, parques arqueológicos, visita a comunidades indígenas). El Aprendizaje Basado en Problemas (De Miguel, 2006) se inscribe en el constructivismo y su aporte consiste en transgredir las formas tradicionales de educación en la medida en que busca que el usuario(a)/estudiante vaya más allá de la situación vertical de trasmisión y comprobación de información del sistema tradicional:

En el aprendizaje constructivo interno no basta con la presentación de la información a la persona para que aprenda, sino que es necesario que la construya o la aprehenda mediante una experiencia interna. El aprendizaje consiste en un proceso de reorganización interno. Desde que se recibe una información hasta que la asimila completamente, la persona pasa por fases en las que modifica sus sucesivos esquemas hasta que comprende plenamente dicha información. La creación de contradicciones o conflictos cognoscitivos, mediante el planteamiento de problemas e hipótesis para su adecuado tratamiento en el proceso de enseñanzaaprendizaje, es una estrategia eficaz para lograr el aprendizaje. El aprendizaje se favorece enormemente con la interacción social. (http://educrea.cl/aprendizaje-basadoen-problemas-el-metodo-abp)

Son tres los ejes del ABP la gestión del conocimiento, la práctica reflexiva y la adaptación a los cambios. En la gestión de conocimientos el usuario es libre de organizar y priorizar los insumos que la aplicación transmedial y los materiales con contenido en realidad aumentada le ofrezcan. Los otros pasos antes analizados de la propuesta de neurodidáctica de Roberto Rosler contribuyen a 
la práctica reflexiva y la adaptación a los cambios. Este enfoque metodológico le da al estudiante un papel más activo en investigar y discernir criterios para encontrar la solución a retos o problemas presentados en la situación educativa. EL ABP es un método que supone una actitud activa de parte del discente. La metodología ABP permite desarrollar diferentes competencias: resolución de problemas, toma de decisiones, Trabajo en equipo, habilidad de comunicación, desarrollo de actitudes y valores.

Esencialmente, la metodología $\mathrm{ABP}$ es una colección de problemas cuidadosamente construidos por grupos de profesores de materias afines que se presentan a pequeños grupos de estudiantes auxiliados por un tutor. Los problemas, generalmente, consisten en una descripción en lenguaje muy sencillo y poco técnico de conjuntos de hechos o fenómenos observables que plantean un reto o una cuestión, es decir, requieren explicación. La tarea del grupo de estudiantes es discutir estos problemas y producir explicaciones tentativas para los fenómenos describiéndolos en términos fundados de procesos, principios o mecanismos relevantes. (Vizcaíno y Suárez, sf p12)

De acuerdo con las autoras mencionadas la metodología ABP tiene los siguientes siete pasos: Aclarar conceptos y términos, definir el problema, analizar el problema, resumen sistemático, formular objetivos de aprendizaje, buscar información adicional fuera del grupo de trabajo o individualmente, síntesis de la información adquirida. La siguiente sección analiza ese camino en el desarrollo de la propuesta Umbrales.

\section{Desarrollo y ejecución}

El Aprendizaje Basado en Problemas también favorece el pensamiento crítico y el tomar conciencia del propio proceso ya que se centra en el trabajo autodirijido o en grupos pequeños donde se planifica. Está compuesto por 7 pasos que a continuación se describen. Con esta perspectiva metodológica se escogió la historia oral del Tesoro de la Isla del Caño como complemento a la experiencia del videojuego UMBRALES siguiendo este razonamiento:

\section{Problema (paso 1) Exposición del usuario (a) a "UMBRALES". Mundo inmmersivo en UNITY $3 D$}

Carlos, el protagonista, es un joven de origen boruca que acompaña a regañadientes a su padre en una celebración de la Fiesta de los Diablitos. Poco interesado en la celebración, explora un museo donde una máscara llama su atención. Al tratar de ponérsela pasa algo mágico y se transporta a Boruca en una época ancestral. Ahí encuentra al cacique Cuasrán quien le explica por qué lo transportó al pasado y que sólo Carlos mismo puede buscar el camino de vuelta a su tiempo. Carlos debe interacturar con otros personajes con quienes intercambia objetos y aprende sobre etnobotánica de la zona, cultura material, tradiciones orales boruca, fauna y flora locales y aprende algunas expresiones y palabras en boruca de alimentos y animales. La única forma de poder regresar a su tiempo es cumplir con todas esas actividades dentro del juego virtual.

Figura 1: Modelización 3D de Carlos

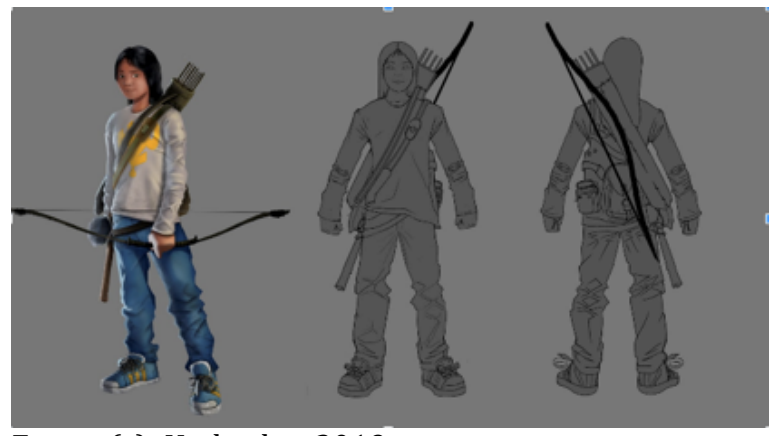

Fuente(s): Umbrales, 2019.

Figura 2: Pantalla de Entrada a Umbrales

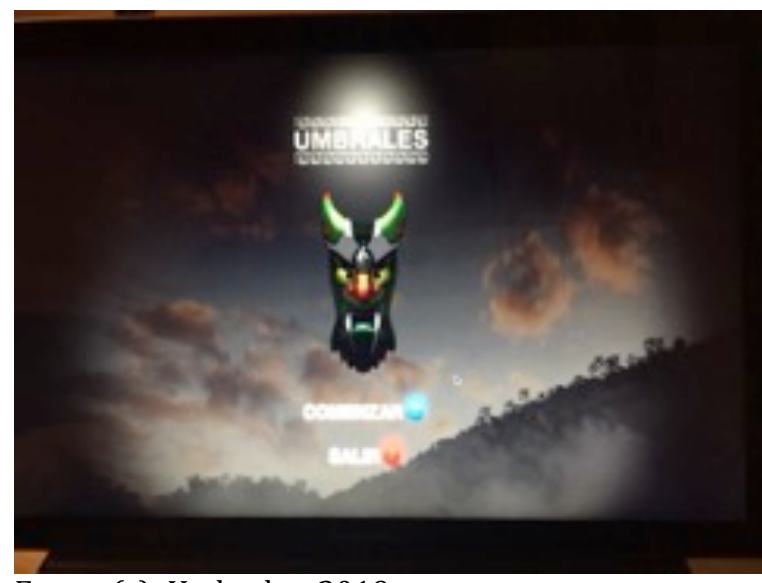

Fuente(s): Umbrales, 2019. 
Figura 3. Carlos frente a la Quebrada Boruca

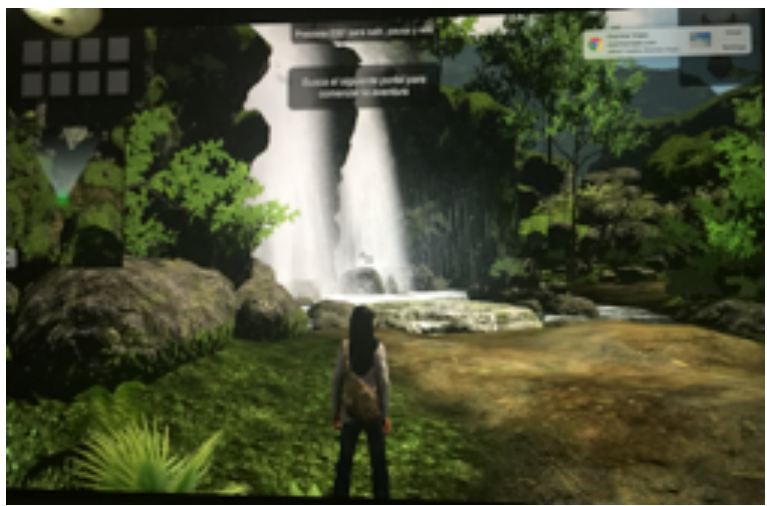

Fuente(s): Umbrales, 2019.

Figura 4. Menú de diálogo de Carlos con Cazador

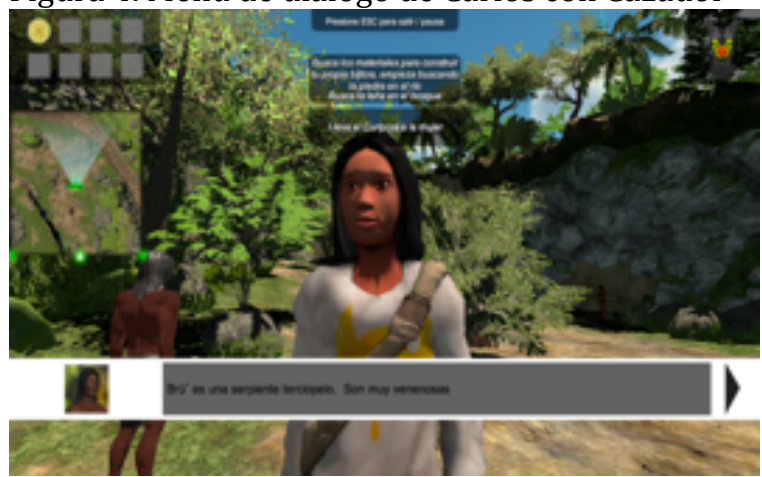

Fuente(s): Umbrales, 2019.

Al finalizar las misiones en Umbrales, Carlos puede regresar al presente, pero su recompensa es una máscara que puede armar como rompecabezas realidad aumentada Originalmente fue diseñada por el artista boruca Kamel González y se digitalizó para producir el rompecabezas. Esa máscara tienen un papel protagonista en el "folleto" Los tesoros de la Isla del Caño" pues le posibilita viajar a otro tiempo: A la época cuando los Boruca viajaban en balsas a la Isla del Caño desde tierra adentro hacia al Pacífico Sur 19 millas mar adentro.
Figura 4.1 Rompecabezas 1

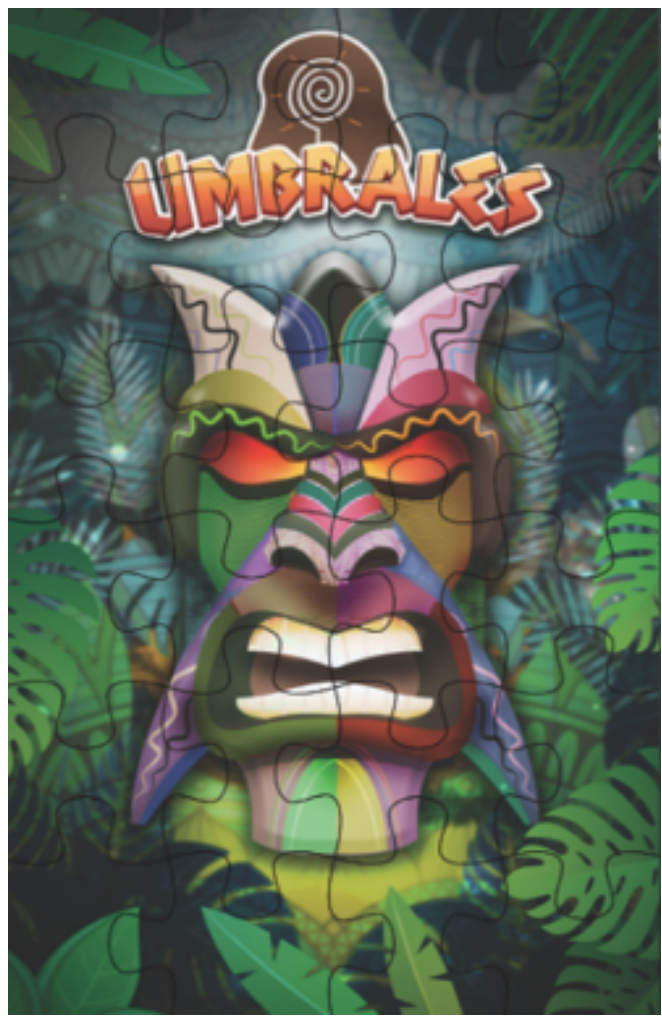

Fuente(s): Umbrales, 2019.

Figura 4.2 Rompecabezas

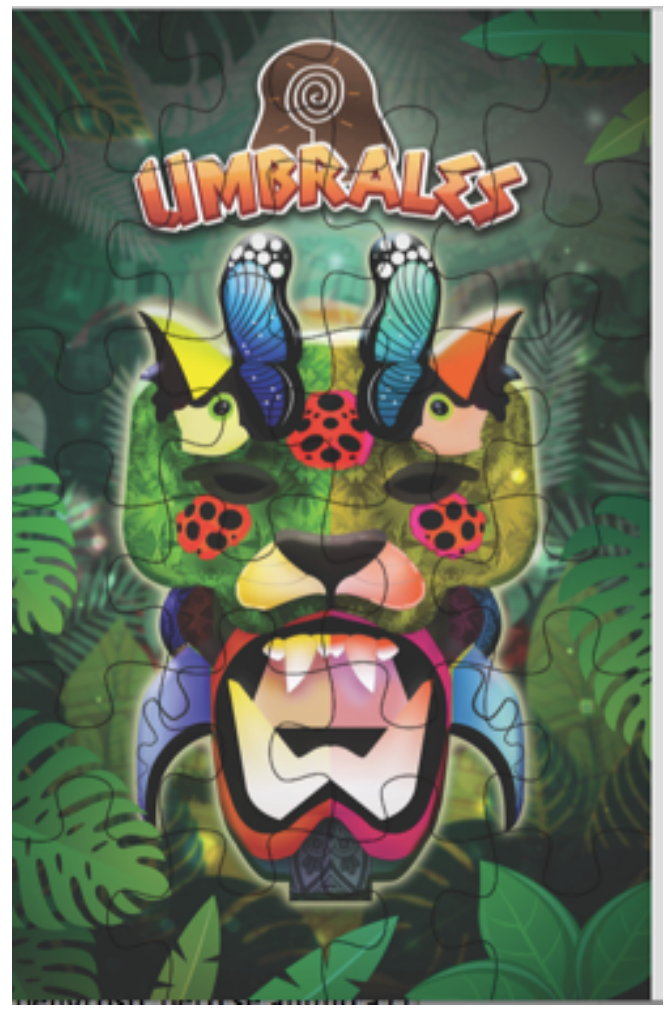

Fuente(s): Umbrales, 2019. 
Umbrales representa aquí la primera fase de un problema doble para el usuario/Carlos: Desde el punto de vista narrativo (debe resolver su regreso al presente) y desde el punto de vista de generación de interés por el patrimono tangible e intangible de los boruca (al hacer el recorrido y hablar con los personajes entrará en contacto con historias y hechos que no conocía o no les daba importancia). Es decir, tanto los usuarios boruca como aquellos que no lo son, se ven enfrentados a un doble problema. Umbrales funciona como una especie de "clase invertida" (flipped class) donde el usuario(a) interactuando con objetos $y$ personajes en el mundo resuelve en un primer paso un problema doble: por un lado cómo avanzar en su vuelta a su tiempo original (nivel narrativo) por otro un problema moral su desinterés/desconocimiento por sus tradiciones e identidad cultura (nivel patrimonio cultural).

Decimos que se trata de una modalidad de clase invertida porque el proceso de enseñanza aprendizaje se hace desde la inmersión en un mundo 3D donde hay misiones concretas que tienen gratificaciones que hacen posible avanzar en la narrativa y esa inmersión se convierte en un espacio de enseñanza-aprendizaje basado en la búsqueda de resolución de misiones.

\section{Discusión previa (pasos 1-5)}

El sitio web despliega guías didácticas para ser usadas en talleres y o clases sobre patri monio boruca y que sirven como espacios entre facilitadores (ya sean docentes o no). Algunos elementos de los materiales didácticos que se elaboraron son: máscaras para colorear. Las máscaras se desgargan y una despliega el rompecabezas en realidad aumentada; la otra una entrevista a su creador que se despliega en un video en realidad aumentada donde Kamel González, explica la importancia de éstas en la cultura boruca.

En cuanto a Los Tesoros de la Isla del Caño, en este folleto se combina texto tradicional, videos submarinos, juegos interactivos, animaciones, juegos de objetos ocultos.

Se diseñaron las guías didácticas para darle seguimiento a este estímulo rico en sonidos, color e interacciones. Siguiendo la metodología ABP, el problema aquí es cómo después de armar satisfactoriamente el folleto, el usuario tiene acceso al libro móvil, lo puede construir él mismo descargando los archivos desde el sitio parallax del proyecto o un facilitador o facilitadora, le puede entregar el folleto ya armado.

Figura 5. Los Tesoros de la Isla del Caño (portada y contratapa)

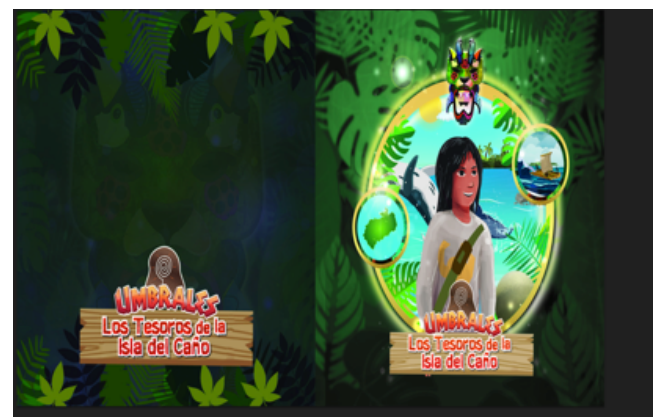

Fuente(s): Umbrales, 2019.

Esta historia la construyeron los investigadores con base en tradiciones orales boruca e investigación básica con historiadores y antropólogos de la Universidad de Costa Rica. La narración inicia cuando Carlos camina con la máscara que obtuvo del cacique Cuasrán, como premio a sus misiones. Camina por el bosque en busca de un petroglifo que abre un umbral en el tiempo para visitar a los indios de río mientras preparan su viaje a la Isla Del Caño. Se une a ellos y empieza la aventura. Interesa resaltar que en esta historia Carlos es solo la excusa, ya aquí los protagonistas son los indios de río, el río mismo, la fauna marina de la bahía de Drake y de la Isla del Caño, de forma secundaria, los piratas que la utilizaron y los grupos indígenas que tenían reliquias y sitios sagrados en el lugar.

Figura 6 Carlos en busca del Umbral señalado por petroglifo

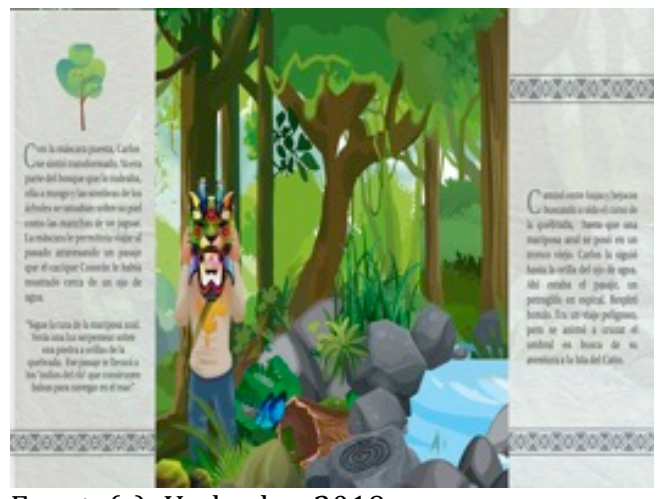

Fuente(s): Umbrales, 2019. 
Figura 7 Carlos en la construcción de balsas de río

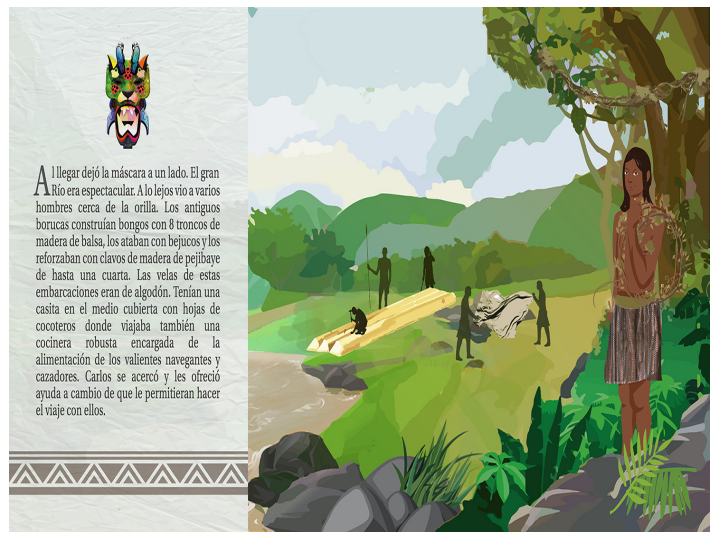

Fuente(s): Umbrales, 2019.

Camino a la isla se hace énfasis en la dificultad y el peligro de la navegación mar adentro, se indican actividades que los borucas desarrollaban ahí También hay videos submarinos de la riqueza marina de la isla del Caño. Se estimula el trabajo en equipo para descubrir qué tipo de fauna y flora marina hay en la zona y se les pide a los usuarios que armen los paper toys que se pueden descargar del sitio web y construyan historias de animales marinos" . La historia de "Los tesoros de la Isla del Caño" también narra las riquezas arqueológicas de la isla y otros aspectos legendarios como piratas o cavernas submarinas con tesoros. Finalmente, hay un juego de objetos ocultos sobre la fauna marina local. De seguido, iniciaría la etapa seis del ABP.

Figura 8. Navegación por el Río

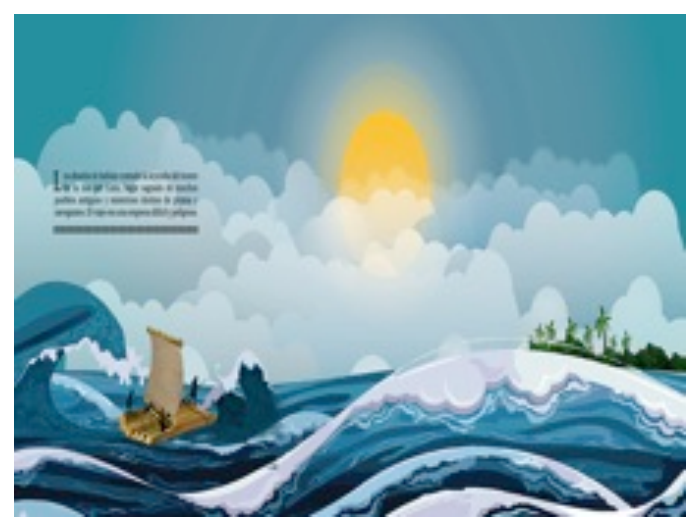

Fuente(s): Umbrales, 2019.

\footnotetext{
${ }^{1}$ Estos materiales son de uso gratuito y se encuentran en un sitio promocionado por Canon titulado Creative Park Recupetado de https://creativepark.canon/en/contents/CNT0010363/index.html
}

Figura 9 Ballenas Jorobadas

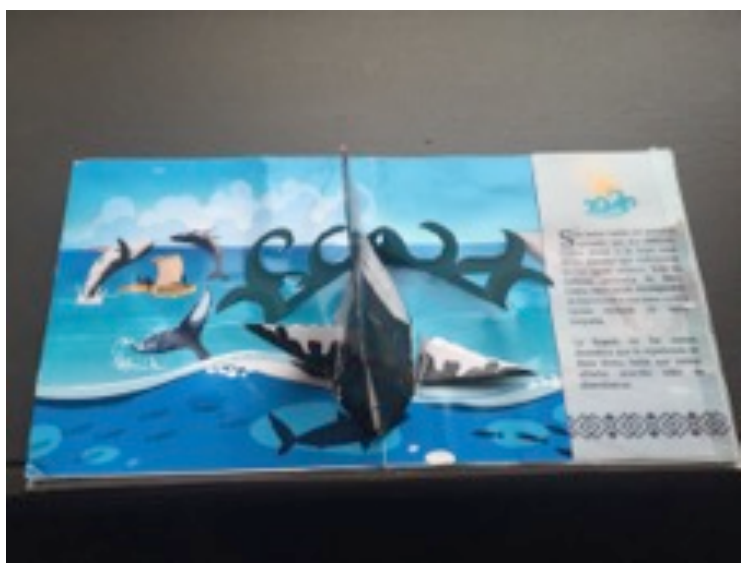

Fuente(s): Umbrales, 2019.

\section{Seguimiento individual (paso 6)}

Como siguiente paso para una educación basada en problemas, los participantes podrán descargar de la página web maestra más materiales para continuar la aventura narrativa y a la vez reforzar valores, costumbres y tradiciones borucas tradicionales. La idea es que esta exploración se dé de forma individual, buscando otras fuentes ya sea bibliográficas, en la red, mediante recolección de historias orales de otros miembros de la comunidad boruca, investigación propia, etc.

Los materiales ofrecidos aquí son plantillas de historietas que los participantes pueden completar tipo "Information gap", es decir, se suprime parte de la información para que sea reconstruida por los participantes con sus propios recursos. El sitio también les aporta enlaces para que los participantes sean capaces de desarrollar sus propias historias sobre otros elementos cubiertos por el cuento (el misterio de las esferas, la riqueza marina, la navegación entre los borucas antiguos, rutas comerciales antiguas y tradiciones orales, los piratas y sus tesoros) y les abre la posibilidad de compartir sus creaciones en la galería del sitio web del proyecto, en Facebook, Snapchat o en otras redes sociales. 
Figura 10 Pirata-Monstruo en Realidad Aumentada defendiendo cueva de tesoros

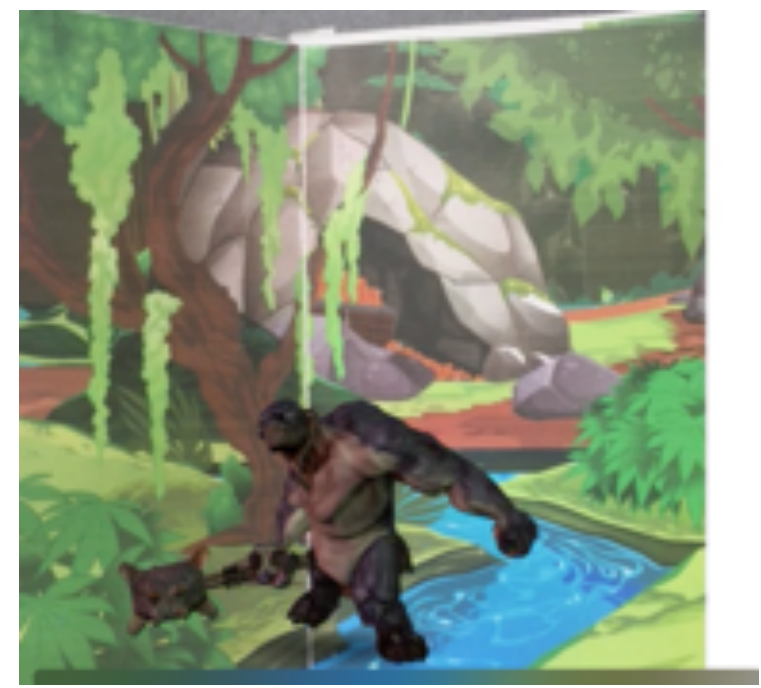

Fuente(s): Umbrales, 2019.

Figura 11 Video Submarino en Realidad Aumentada

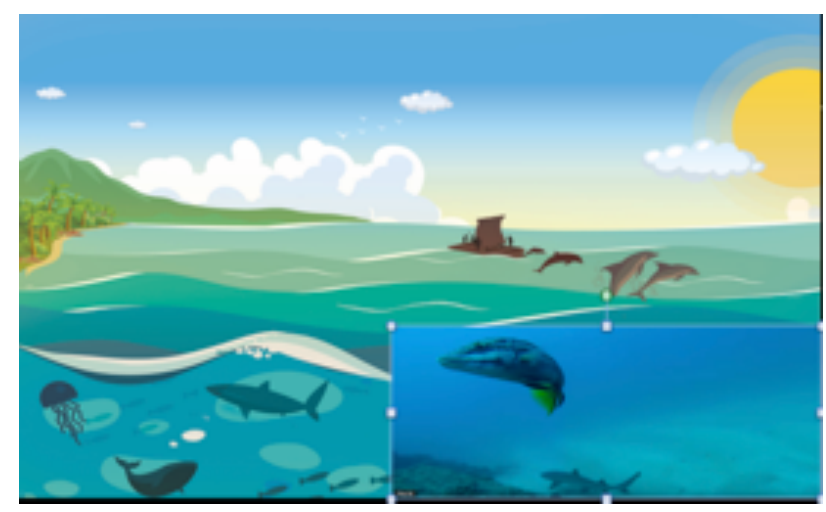

Fuente(s): Umbrales, 2019.

Figura 12 Tigrillo

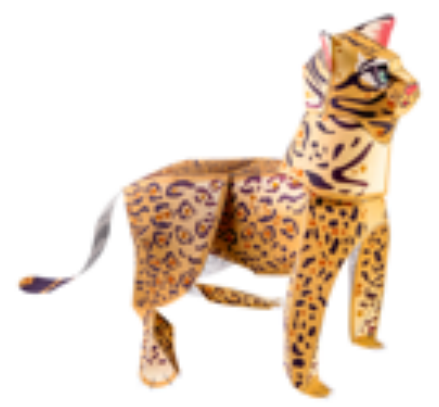

Fuente(s): Umbrales, 2019.
Figura 13 Rana de Arbol

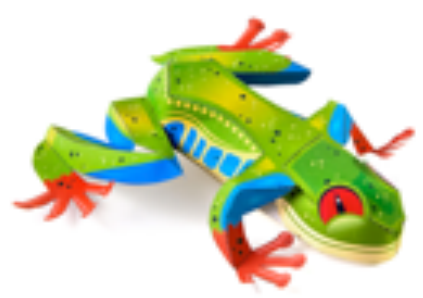

Fuente(s): Umbrales, 2019.

Figura 14 Tucán

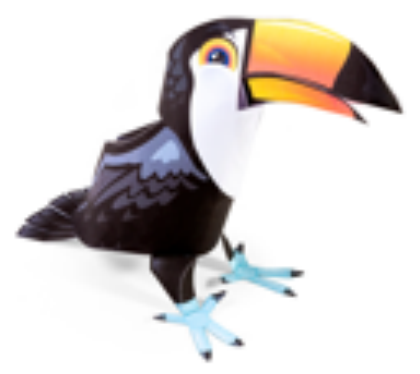

Fuente(s): Umbrales, 2019.

Como el relato está relacionado con navegación y el mar, se le ofrecen a los participantes varias posibilidades temáticas para explorar más aspectos de la cultura boruca.

1. Proponer recorridos históricos o legendarios de la zona (geolocalización en Google Earth o google Maps asociada a fotos del lugar).

2. Hacer historias de piratas y la Isla del Caño (escritura creativa).

3. Proponer una caricatura o animación para explicar cómo los boruca obtenían sal marina (se le dan los enlaces con las herramientas y ejemplos. Se sugiere el uso de Scratch, CoSpaces, o Alice 3D).

4. Contar con tiras cómicas otras leyendas boruca asociadas al mar (Los Brobroyeras y la sirena Unquí so, enseñarles cómo digitalizarlas y compartirlas).

5. Hacer narrativas interactivas usando herramientas digitales que pueden ir exponiendo en la galería del sitio.

Con esta parte el proyecto lograría ser verdaderamente transmedial al incorporar la creación de contenido creado por los usuarios. 


\section{Seguimiento posterior (paso 7)}

Finalmente, en el último paso se le ofrece a los participantes descargar del sitio web guías didácticas que le permitan verificar si el problema inicial:

- Se resolvió.

- Se mejoró el conocimiento sobre los borucas.

- Se generó conocimiento nuevo.

- Se cambió la actitud hacia el patrimonio tangible e intangible boruca.

- Proponer que Carlos viaje a otra época porque aún no ha aprendido suficiente.

\section{Resultados}

Los principales resultados de grupos focales con usuarios potenciales se derivaron de tres grupos focales. Los participantes se reclutaron a conveniencia y cursaban entonces el primer ciclo de primaria en escuelas privadas y públicas de Costa Rica. La técnica fue muestreo por bola de nieve. En los primeros dos grupos focales participaron 13 individuos 7 varones : 4 del área metropolitana y tres de la provincia) y 6 mujeres : 3 del area área metropolitana y 3 de provincia, a quienes se les hizo un pretest ante de la exposición a la experiencia transmedia, luego se realizó un focus group informal o estilo inglés donde la investigadora les hizo algunas preguntas sobre el juego mientras comían pizza y una semana después del estímulo un posttest telefónico para comprobar recordación y aprendizaje de contenidos sobre la cultura Boruca. Un tercer grupo focal (7 individuos 3 varones y 4 mujeres) fue expuesto solamente a la historia "Los tesoros de la isla del Caño", solo como texto escrito no como libro ilustrado con elementos de ingeniería de papel y elementos de realidad aumentada. Esto con el fin de determinar aspectos de recordación de la historia "Los tesoros dela isla del Caño" y compararlo con los de los grupos expuestos a toda la experiencia transmedia Los principales hallazgos han sido satisfactorios y se enumeran a continuación:

- Los usuarios disfrutan del diseño meticuloso del ambiente. De los movimientos de Carlos al saltar o caminar. Se asombran por la calidad gráfica.

- Un usuario boruca (adulto) a quien se le mostró UMBRALES, indica que Cuasrán debe ser más viejito, pero eso da pie para conversaciones extrajuego de quién y cómo es el cacique Cuasrán. Hasta puede derivarse en una guía didáctica complementaria a Umbrales donde los usuarios dibujen a Cuasrán tal y como lo imaginan.

- La introducción es corta y algunos usuarios tienden a saltársela, de todas maneras, aún así las pruebas de pretest y postest de conocimientos sobre los boruca indican que estar expuesto a Umbrales siempre resulta en un mayor conocimiento de tradiciones y lengua boruca y genera interés de parte de los usuarios sobre querer continuar la historia.

- El juego resultante se juega en menos de 10 minutos por lo que lo hace perfecto para estar en espacios de exhibición como salas de museos. Generalmente los usuarios, dependiendo de cuán "gamers" sean logran terminar el juego entre $10 \mathrm{y}$ 15 minutos. Luego de jugarlo manifestaron que les gustaría aprender más sobre el juego.

Los menú de diálogos al principio no son muy comprensibles, especialmente cómo avanzar en ellos, pero una vez que se dan cuenta de cómo proseguir no enfrentan problema alguno.

A una semana de haber sido expuestos a UMBRALES los usuarios recuerdan el nombre de Carlos, algunas palabras en boruca de frutas y animales, el nombre del cacique Cuasrán y la historia del Rey de los Chanchos. Además queda la idea de que hay una planta que se come y que es también medicinal, pero solo dos usuarios recordaron su nombre en boruca.

- A veces el personaje de Carlos puede caer entre algunas piedras y no se lo puede sacar a menos de que se reinicie el juego. Es inevitable porque Carlos tiene libre acceso al espacio desarrollado.

- De los usuarios que vieron la introducción (solo 4, la mayoría se salta la introducción) dos se quejan de que 
Carlos en el museo primero es más moreno y luego parece una niña.

\section{Conclusiones}

A lo largo de este proceso se concluye y recomienda lo siguiente:

Es fundamental contar con el acompañamiento y aval de miembros de la comunidad boruca para que el proceso de diseño, la validación y la implementación puedan darse de manera efectiva.

La implementación de tecnologías innovadoras en procesos de enseñanza aprendizaje no es un fin en sí misma, también se debe acompañar a los usuarios ya sea con guías didácticas $o$ con el entrenamiento de facilitadores.

Es vital para la academia seguir experimentando y potenciado las posibilidades de las tecnologías emergentes (realidad virtual, realidad aumentada o realidad mixta y extendida) en procesos de enseñanza aprendizaje.
Proyectos de este tipo deben visualizarse a largo plazo, pues en su proceso de desarrollo, se hace necesario actualizar y mejorar diversos aspectos tanto de contenido ( al validarlo con las comunidades originarias) como de experiencia de usuario. Es una ventaja de este tipo de herramientas y un reto que como academia debemos visualizar a la luz de la vigencia de este tipo de proyectos.

Las estrategias transmedia son experiencias que permiten un aprendizaje crítico e inclusivo, ya que se fomenta el aprendizaje desde diferentes habilidades, competencias, narrativas que promueven el aprendizaje colaborativo.

Desde una pedagogía decolonial y una propuesta de educación intercultural es posible desaprender las categorías racializadas $y$ excluyentes de muchas propuestas escolares tradicionales.

Umbrales como propuesta transmedia permite visibilizar formas alternativas de conocer e interactuar con los otros, con el ambiente que nos rodea para alcanzar un bienestar común basado en el respeto y la diversidad. 
UMBRALES: Gamificación para la Educación Intercultural

\section{Referencias}

Austakaldis, S. (2017) Practical Augmented Reality: A guide to the Technologies, Applications and Human Factors for AR and VR (Usability). Indiana: RR Donelley.

Baker, C. (2004). The Sage Dictionary of Cultural Studies. London: Sage

Chiang, T. H. C., Yang, S. J. H., \& Hwang, G. H. (2014). Students online interactive patterns in augmented reality-based inquiry activities. Computers \& Education, 78, pp. 97-108

Constenla, A. (2011) Leyendas y Tradiciones Boruca. Tomo 1. San José: EUCR

De Miguel, M. (2006). Metodologías de enseñanza y aprendizaje para el desarrollo de competencias. Orientaciones para el profesorado universitario ante el espacio europeo de educación superior. Recuperado de http://gio.ascis.net/wp-content/uploads/2010/05/Lecturas-2.-Papers-yPublicaciones-de-estrategais-de-aprendizaje.doc

De Sousa Santos B. (2009). Una epistemología del Sur. Buenos Aires: Clacso.

De Sousa Santos, B. y Meneses, M.P. (2014). Epistemologías del Sur. Perspectivas. Madrid: Akal.

Fonseca, O. (2012). Historia Antigua de Costa Rica. Surgimiento y caracterización de la primera civilización. San José: EUCR

Garro Valverde, L. (2010). Saberes y Sabores Boruca. San José: Gamaprint.

Lanham, M. (2017). Augmented Reality Game Development. Edición Kindle.

Montero, M. (2019). Países de Latinomérica con más líneas de telefonía móvil. En Trece Bits. Redes Sociales y tecnología. 18 de febrero. Recuperado de https://www.trecebits.com/2019/02/18/latinoamerica-telefonia-movil/

Morales, E.S. (1999) Lengua o Dialecto Boruca o Brúnkajk. Rcopilado por Miguel Angel Quesada Pacheco. San José: EUCR.

Morales, P. Y V. Landa. (2004). Aprendizaje basado en problemas. Theoria, 13, pp. 145-157. Recuperado de http://redalyc.uaemex.mx/redalyc/pdf/299/29901314.pdf

Quesada Pacheco, M.A. (1996). Narraciones Borucas. San José: EUCR.

Quintanilla, I. (2002). Moluscos, tintes y textiles: historia del uso del tinte morado entre las artesanas borucas de Costa Rica. Actas de las II Jornadas Internacionales sobre textiles precolombinos (V. Solanilla, ed). Universitat Autònoma de Barcelona-Institut Catalá de Cooperación Iberoamericana, Barcelona, pp:43-59.

- (2004). La técnica de teñido directo con caracoles: el ejemplo de los boruca de Costa Rica. PURPURAE VESTES. I Simposium Internacional sobre Textiles y Tintes del Mediterráneo en época Romana (C. Alfaro, J.P. Wild y B. Costa, eds.), España, pp.245-252.

- (2012) Los boruca y el tejido con caracoles marinos. Recuperado de https://ifigeniaquintanilla.com/2012/05/23/los-boruca-y-el-tenido-de-algodon-con-caracolesmarinos/ 23 de mayo.

Scolari, C. (2013). Narrativas Transmedia. Cuando todos los medios cuentan. Barcelona: Deusto-Planeta.

- (2015). Ecología de los medios. Entornos, evoluciones e interpretaciones. Barcelona: Gedisa.

- (2018). Alfabetismo transmedia en la nueva ecología de los medios. Libro blanco (Enero). Recuperado de http://www.codajic.org/node/3027

Stone, D. (1949). Los boruca en Costa Rica. Paper of Peabody Museum of American Archeology and Ethnology. San José, Costa Rica: Centro de Investigación y Conservación de Patrimonio Cultural. Ministerio de Cultura y Juventud.

Tubino, F. (2005). La interculturalidad crítica como proyecto ético-político. En Encuentro continental de educadores agustinos. Lima, 24-28 de enero de 2005. Recuperado de http://oala.villanova.edu/congresos/educación/lima-ponen-02.html

Vizcaíno, C. y Juárez, E. (sf) ¿Qué es y cómo funciona el aprendizaje basado en problemas? En $L a$ Metodología del ABP. Recuperado de http://www.ub.edu/dikasteia/LIBRO_MURCIA.pdf

Walsh, C. (2014). Interculturalidad crítica y pedagogía decolonial: Apuestas (Des)de el in-surgir, re-existir, re-vivir. Simposio Internacional: El significado de la negritud/ el significado de ser negro. Retrieved 
Revista Internacional de Educación y Aprendizaje, 8(1), 2020, pp. 57-72

from: $\quad$ https://redinterculturalidad.wordpress.com/2014/02/06/interculturalidad-critica-ypedagogia-decolonial-catherine-walsh/comment-page-1/

Walsh, C. (2009). Interculturalidad crítica y educación intercultural. Recuperado de www.uchile.cl/.../interculturalidad-critica-y-educacion-intercultural.pdf 